\title{
The brain signature of paracetamol in healthy volunteers: a double-blind randomized trial
}

This article was published in the following Dove Press journal:

Drug Design, Development and Therapy

23 July 2015

Number of times this article has been viewed

\author{
Gisèle Pickering ${ }^{1-3}$ \\ Adrian Kastler ${ }^{4}$ \\ Nicolas Macian ${ }^{1,2}$ \\ Bruno Pereira ${ }^{5}$ \\ Romain Valabrègue ${ }^{6}$ \\ Stéphane Lehericy ${ }^{6}$ \\ Louis Boyer ${ }^{4,7}$ \\ Claude Dubray ${ }^{1-3}$ \\ Betty Jean ${ }^{4}$ \\ 'CHU Clermont-Ferrand, Centre \\ de Pharmacologie Clinique, ${ }^{2}$ Centre \\ d'Investigation Clinique - Inserm \\ 1405, ${ }^{3}$ Clermont Université, \\ Laboratoire de Pharmacologie, \\ Faculté de médecine, ${ }^{4} \mathrm{CHU}$ Gabriel \\ Montpied, Clermont-Ferrand, \\ Service d'Imagerie Ostéo-articulaire \\ thoracique et neurologique, ${ }^{5} \mathrm{CHU}$ \\ Clermont-Ferrand, Délégation \\ Recherche Clinique et à l'Innovation, \\ Clermont-Ferrand, France; ${ }^{6}$ nstitut \\ du Cerveau et de la Moelle epiniere \\ - ICM, Centre de Neurolmagerie \\ de Recherche CENIR, Inserm \\ UII27, CNRS UMR 7225, Sorbonne \\ Universités, UPMC University \\ Paris, Paris, France, Department of \\ Neuroradiology, Groupe Hospitalier \\ Pitié-Salpêtrière, Paris, France; ${ }^{7} U M R$ \\ CNRS UdA 6284, Clemont-Ferrand, \\ France
}

Correspondence: Gisèle Pickering Clinical Pharmacology Centre,

Bâtiment 3C, CHU of Clermont-Ferrand, 58 Montalembert Road, 6300 I ClermontFerrand cedex, France

$\mathrm{Tel}+33473178416$

Fax +33473178412

Email gisele.pickering@udamail.fr
Background: Paracetamol's (APAP) mechanism of action suggests the implication of supraspinal structures but no neuroimaging study has been performed in humans.

Methods and results: This randomized, double-blind, crossover, placebo-controlled trial in 17 healthy volunteers (NCT01562704) aimed to evaluate how APAP modulates pain-evoked functional magnetic resonance imaging signals. We used behavioral measures and functional magnetic resonance imaging to investigate the response to experimental thermal stimuli with APAP or placebo administration. Region-of-interest analysis revealed that activity in response to noxious stimulation diminished with APAP compared to placebo in prefrontal cortices, insula, thalami, anterior cingulate cortex, and periaqueductal gray matter.

Conclusion: These findings suggest an inhibitory effect of APAP on spinothalamic tracts leading to a decreased activation of higher structures, and a top-down influence on descending inhibition. Further binding and connectivity studies are needed to evaluate how APAP modulates pain, especially in the context of repeated administration to patients with pain.

Keywords: paracetamol, nociception, fMRI, pharmacology, pain

\section{What is really known about this subject?}

The mechanism of action of paracetamol (APAP) is complex and a central mechanism of action has been described to explain its analgesic effect. However, this has not been to date visualized by neuroimaging and brain areas involved in the analgesic effect of APAP have not been studied.

\section{What this study adds?}

APAP 1) decreases activation of higher structures involved in pain and in cognitive/ affective processing and 2) exerts a top-down influence on descending inhibitory pathways and midbrain neurotransmission. This functional magnetic resonance imaging (fMRI) study opens interesting avenues for research on the mechanism of action of APAP per se and beyond that, for the development of new non-opioid analgesics.

\section{Introduction}

APAP (acetaminophen), one of the most frequently prescribed analgesics worldwide, has been largely explored in preclinical, molecular, and clinical studies over the last 2 decades. The findings of its central mechanism of action, ${ }^{1-4}$ the concept of APAP as a prodrug with analgesic APAP metabolites, 5,6 the implication of serotonergic, opioidergic, vanilloid, cannabinoid receptors as well as calcium channels ${ }^{7-13}$ have transformed our view of APAP from a drug acting at the periphery to a complex molecule with spinal and supraspinal actions. Clinical studies in healthy volunteers have confirmed the central serotonergic activation ${ }^{4}$ and the reinforcement by APAP of descending inhibitory pain 
pathways $^{3}$ in order to obtain APAP analgesic effect. This pain inhibitory system comprises a network of cortical regions and brain stem nuclei, the periaqueductal gray matter (PAG) and the rostral ventromedial medulla (RVM) that project to the dorsal horn of the spinal cord. ${ }^{14-16}$ Both the PAG and RVM receive direct projections from the spinal dorsal horn and may control the ascending nociceptive input by a feedback mechanism. ${ }^{17,18}$ In the few missing pieces of the APAP mechanism of action puzzle, so far there has been a limited number of neuroimaging studies with APAP in humans.

fMRI allows to elucidate pharmacodynamic effects of a drug on brain function by combining the administration of a drug with fMRI. A number of studies with analgesics have demonstrated the coupling between subjective pain intensity ratings and objective blood oxygen level-dependent (BOLD) responses measured in central structures. ${ }^{19-21}$ The brain regions found active with $\mathrm{fMRI}$ during an acute physical pain experience, the so-called "pain matrix", are well documented. ${ }^{16}$ As pain is a subjective experience, ${ }^{22-24}$ some of these regions (dorsal anterior cingulate cortex [ACC], anterior insula) are also involved in the affective and unpleasant component of acute physical pain, as well as in social pain. ${ }^{25,26}$ Among analgesics, opioids, ${ }^{27-32}$ non steroidal antiinflammatory drugs, aspirin and parecoxib ${ }^{33}$ and ketamine ${ }^{34}$ have been studied by fMRI.

Considering the corpus of research on APAP pharmacology at the central level and the fact that the cerebral signature of APAP during physical pain has not been studied yet, this fMRI trial has been designed and combines physical pain induction with APAP administration. This clinical trial in healthy volunteers aims to evaluate how APAP modulates pain-evoked fMRI signals and how cerebral structures of the pain matrix and the PAG, cornerstone of APAP mechanism of action, are involved in APAP antinociception.

\section{Methods}

\section{Study protocol}

This randomized, double-blind, crossover, controlled trial in healthy volunteers took place in the Clinical Investigation Centre/Clinical Pharmacology Centre and in the Neuroimaging Department of the University Hospital of ClermontFerrand, France, between January 9, 2012 and April 13, 2012. The study was reviewed and approved by the French Institutional Review Board and by the French Drug Agency. It followed standardized ethical and safety Good Clinical Practice Guidelines, and procedures were in accordance with the Declaration of Helsinki. It was declared on clinialtrials. gov (NCT01562704).

\section{Volunteers}

Participants were recruited through the database of the Clinical Pharmacology Centre of the University Hospital of Clermont-Ferrand, France. Male volunteers (to avoid pain thresholds variability due to menstrual cycle in females) were eligible if they were $\geq 18$ years old, did not take analgesic or anti-inflammatory treatment in the last 7 days and were nonsmokers. Exclusion criteria included the following: a known hypersensitivity to APAP, concomitant pathologies, contraindications in the realization of the MRI without injection (claustrophobia, pacemaker, hearing aid, cerebral clip etc), consumption of alcohol and addiction to street drugs. Eligible volunteers were informed about the protocol and provided a signed informed consent.

\section{Study design}

A detailed overview of the experimental design used for the fMRI experiments is given in Figure 1. Prior to fMRI experiments, the volunteers were familiarized (two sessions at 1-week interval) with the thermal stimulation and the fMRI procedures. The trial itself consisted of two randomized fMRI sessions 1 week apart, with APAP or placebo according to the randomization list established beforehand by a research assistant who was not involved in the trial. On the day of the experiment, volunteers were comfortably lying on the examination table of the MRI scanner with the thermode strapped on the dominant hand for thermal tests. The pain threshold, defined as the temperature where the stimulation becomes painful, was evaluated and the noxious stimulation temperature (ST) was defined as the pain threshold $+3^{\circ} \mathrm{C}$ according to previous tests performed in our department. Baseline fMRI was recorded using the thermal stimulation paradigm (described later). Each MRI session lasted 40 minutes. Subjects were then randomized at T0 in APAP or placebo groups. Double-blinding was fully respected with the volunteers and the members of staff. A research nurse who was only involved in drug allocation was in charge of drug administration. fMRI data acquisition was then repeated at $\mathrm{T} 0+100$ minutes $(\mathrm{T} 0+100)$ during the application of the same thermal stimulation paradigm. Perceived pain was evaluated at the end of each fMRI session, at baseline and $\mathrm{T}+100$ with a numeric pain rating scale ranging from $0-$ no pain to 10-maximal pain. Volunteers were then discharged after a clinical examination and came back a week later for the second period of the fMRI trial and were administered APAP or placebo in a crossover design.

The double-blind, randomized, and crossover design of this study was chosen in order to be able to discard all 


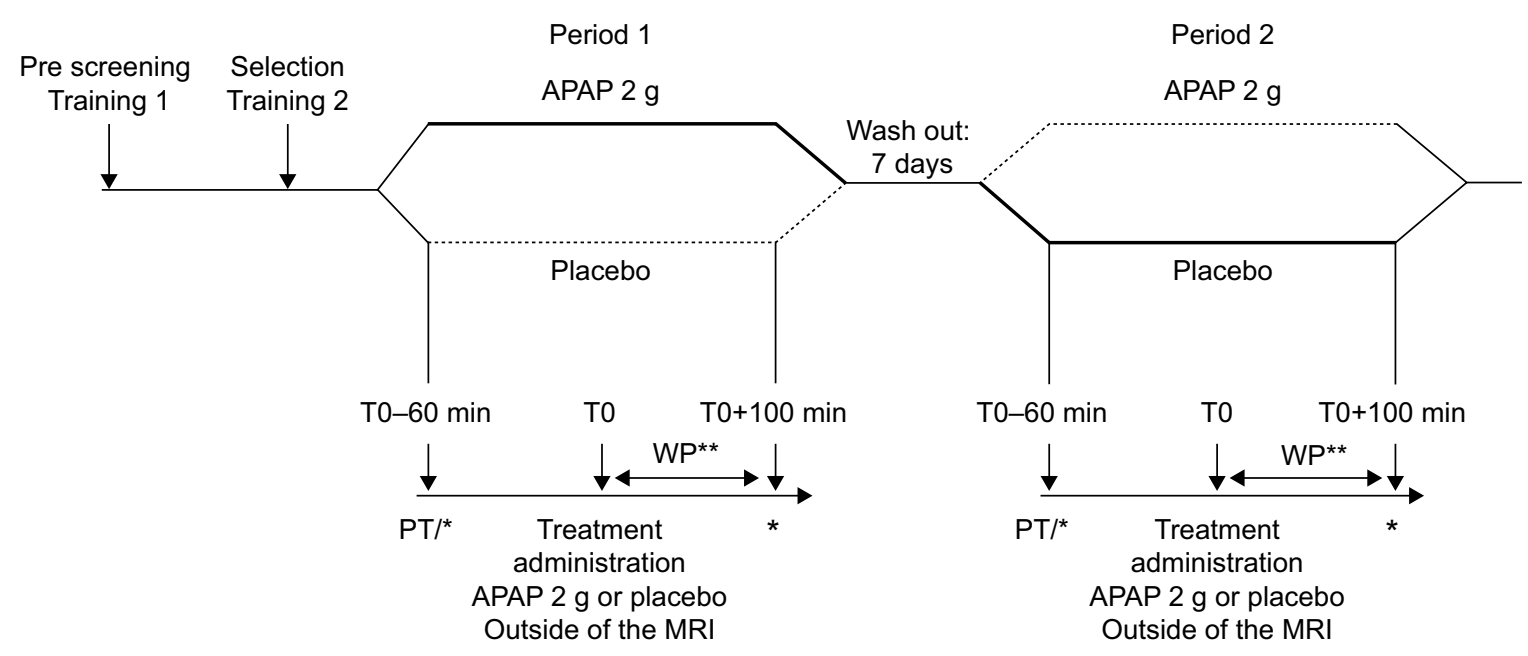

Figure I Chronology of the study.

Notes: PT: determination of pain threshold with Thermotest pathway (Medoc Ltd, Ramat Yishai, Israel). *Pain stimulation following the pain paradigm with Thermotest pathway (Medoc) and acquisition of data with fMRI 3 Tesla (recording session duration: 40 minutes). Waiting period** outside from the MRI.

Abbreviations: APAP, paracetamol; min, minute(s); fMRI, functional magnetic resonance imaging; MRI, magnetic resonance imaging.

variable environmental factors, placebo analgesia, and have the subjects as their own controls. Such a clinical trial will isolate pure drug effects if expectations and beliefs of the subject are identical at each session to avoid any bias. The second test after administration of APAP was set at $\mathrm{T} 0+100$ based on published data on oral APAP with a maximal analgesic effect at 90-100 minutes ${ }^{3}$ and also to allow the patient some time out of the MRI scanner to limit fatigue and poor compliance. The main outcome of the study was the impact of APAP compared to placebo on BOLD fMRI activity by the analysis of the regions-ofinterest (ROI).

\section{Randomization}

Patients were randomized in the APAP group ( $2 \mathrm{~g}$ of oral APAP in four capsules of $500 \mathrm{mg}$ APAP each) or in the placebo group (four capsules of $500 \mathrm{mg}$ lactose each). Randomization was done according to the randomization list that had been established beforehand by a clinical research assistant who was not on site and not involved in the trial. Double-blinding was fully respected. Treatments were prepared in the Central Pharmacy of the University Hospital with double-blinding according to Good Pharmaceutical Practice guidelines. APAP and placebo looked alike (red and white capsules). Randomization was done by blocks of four but this information was not known by the team.

\section{Evoked thermal pain stimulation}

Thermal stimulation was delivered using a $25 \times 25$ Medoc TSA-II thermal sensory analyzer, contact heat-evoked potential stimulator (Medoc Ltd, Ramat Yishai, Israel). ${ }^{35,36}$
An event-related protocol was used to apply contact heat stimuli to the thenar of the right hand (dominant hand, all volunteers were right-handed) during simultaneous fMRI acquisition. The thermode was comfortably applied on the thenar to avoid artifacts when moving the thermode during measurements. The thermode remained at the same site during each block of stimulation and no sensitization of the hand was observed in the feasibility study we carried out before the trial.

The stimulation paradigm (Figure 2) consisted of 20 cycles with 5-second stimuli at ST (ST had been determined beforehand for each volunteer at the start of each period) followed by 15 seconds at the temperature of $32^{\circ} \mathrm{C}$ for a global duration of 8 minutes.

\section{fMRI data acquisition}

fMRI experiments were carried out on a General Electric Discovery MR 750 3.0 T using a 32-channel head coil with subjects lying supine. Initial localizer images were acquired in three planes as a reference for slice positioning for

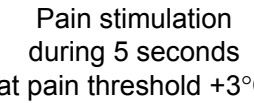

at pain threshold $+3^{\circ} \mathrm{C}$

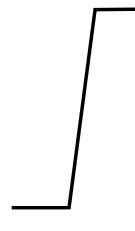

Return to baseline temperature $\left(32^{\circ} \mathrm{C}\right)$ during 24 seconds
Pain stimulation during 5 seconds at pain threshold $+3^{\circ} \mathrm{C}$
Figure 2 Thermal pain paradigm used in the fMRI trial. Abbreviation: $\mathrm{fMRI}$, functional magnetic resonance imaging. 
subsequent fMRI studies. A standard whole-brain gradient echo planar imaging sequence was used for the functional scans (repetition time $=3,000 \mathrm{~ms}$; echo time $=30 \mathrm{~ms} ; 160$ volumes field of view $=240 \times 240 \mathrm{~mm}^{2}$, matrix $=128 \times 128$, voxel size $=1.8 \times 1.8 \times 3.6 \mathrm{~mm}^{3}$, acquisition time $=8$ minutes $)$. A highresolution volumetric three-dimensional (3D) T1-weighted acquisition was performed, for anatomical overlay of activation, in the same session as the functional scans: 3D brain volume imaging (repetition time $=9.96 \mathrm{~ms}$, echo time $=4.1 \mathrm{~ms}$, inversion time $=400 \mathrm{~ms}$, field of view $=240 \times 240 \mathrm{~mm}^{2}$, matrix $=512 \times 512$, voxel size $=1 \times 1 \times 1.2 \mathrm{~mm}^{3}$, acquisition time $=3$ minutes 22 seconds). To achieve synchronization, the trigger output of the scanner was used to initialize the fMRI paradigm and triggers from contact heat-evoked potential stimulation and the scanner were recorded together. fMRI sequences were assessed in the following order: anatomical scout, 3D brain volume, fMRI BOLD sequence echo planar imaging (thermal stimulation).

\section{fMRI data analysis}

The neuroimaging data were preprocessed and analyzed using SPM8 (Statistical Parametric Mapping, Version 8; Wellcome Department of Imaging Neuroscience, London, UK), in Matlab 7.12 (MathWorks). Co-registration of T1 anatomical images was performed prior to spatial normalization into the MNI space. Functional images were first preprocessed with slice-time correction, and motion correction by realigning all images to the first image. The motion correction parameters were examined for each individual and instantaneous movement was always less than a third of the voxel size. The BOLD images were then spatially normalized into the MNI space using trilinear interpolation, with the normalization parameters determined during the normalization of the structural images. Subsequent spatial smoothing using isotropic $8 \mathrm{~mm}$ full width at half maximum Gaussian kernel was applied to the functional images to increase signal-to-noise ratio. A high pass temporal filter (cut-off 128 seconds) and correction for autocorrelation between successive scans were applied to the time series (AR1). On the basis of a priori hypotheses regarding the involvement of the ACC, insula, prefrontal cortices, thalamus, and PAG in the mechanism of action of APAP, we utilized structurally defined ROI analyses to examine between group differences in neural activity in these regions during thermal pain stimulus.

For ROI extraction and beta analysis, we employed a block design with two conditions (baseline and stimulus). To determine those brain regions encoding pain intensity, we performed a whole-brain second-level statistical parametric maps of the $t$ statistic (SPM $\{\mathrm{t}\}$ ) analysis including all subjects and all functional scans obtained at baseline for both examinations (weeks 1 and 2), prior to drug intake. This allowed both to validate baseline pain matrix activation and to define the ROI in which beta extraction would be performed. The following ROI were defined using the Marsbar tool in SPM8: PAG, ACC, insula, prefrontal cortices, and thalami. Beta extractions were then performed with the contrast stimulation-baseline, in order to assess group differences in neural activity in these regions during thermal noxious stimulus. Beta extraction corresponds to the value of the contrast in a selected ROI. It therefore corresponds to the estimate of the stimulus response compared to resting state, as it is an estimation of the effect of the BOLD signal in the ROI. Performing a beta extraction in a selected ROI allows to obtain an averaged beta value for the entire ROI. This extraction method allows to reduce the noise signal and therefore leads to an increase of the signal-to-noise ratio that facilitates stimulus response detection.

\section{Statistical analysis}

Previous pilot fMRI studies with analgesics, opioids, and non-opioids include a variable number of subjects. ${ }^{32,33,37}$ A double-blind, crossover, controlled fMRI study with nonopioids included 14 subjects $^{27}$ and an APAP pharmacological study with cerebral-evoked potentials included 12 subjects. ${ }^{35}$ Considering these data, the estimated number of subjects was 16 , and taking into account potential dropouts, 20 patients had to be included.

Statistical analysis was performed using Stata software, version 13 (StataCorp LP, College Station, TX, USA). The tests were two-sided, with a type I error set at $\alpha=0.05$. The characteristics of patients between groups were compared using Student's $t$-test or Mann-Whitney test according to $t$-test hypotheses (assumption of normality studied by Shapiro-Wilk test and homoscedasticity by Fisher-Snedecor test) for quantitative parameters and using Fisher's exact test for categorical factors. Beta values, presented as the mean \pm associated standard deviation, were compared between groups using random-effects models for crossover designs. These models used the delta values as the dependent variables and included period, group, sequence, ROI, and possible carryover as fixed effects. In these models, we always considered random subject effects (random intercept and slope). The residual normality was checked for all models presented in this article. Random-effect models were performed to consider between and within subject variability (several 
ROI measures for each subject) rather than averaging fMRI signals from different ROI. These models allowed to study the correlation between fMRI signals and the pain intensity considering pain intensity as a fixed effect.

BOLD response amplitude and perceived pain intensity were tested for a significant decrease between placebo and APAP sessions and between the two portions of the experiment, baseline and drug administration. Significance was tested with a paired one-tailed Student's $t$-test at $P<0.05$. BOLD response amplitudes to each stimulus were pooled (averaged) across subjects to achieve adequate signal-tonoise for the parameter estimation.

\section{Results}

Twenty healthy right-handed males were eligible, three did not meet the inclusion criteria and $17(22.7 \pm 3.2$ years old $)$ were randomized and analyzed. The flowchart is presented in Figure 3.

\section{Psychophysics}

The ST was not significantly different between both sessions $\left(47.1^{\circ} \mathrm{C} \pm 2.1{ }^{\circ} \mathrm{C}\right.$ APAP, $46.3 \pm 2.4$ placebo). During the overall fMRI experiment, APAP did modulate significantly $(P<0.001)$ the mean pain ratings on the numerical scale evoked by the thermal stimulation (T0: 6.0土1.2; T100: $3.6 \pm 0.8$ ) compared to placebo that did not diminish pain (T0: 5.8 \pm 1.1 ; T100: 5.6 \pm 1.1 ). APAP reduced the behavioral pain score from moderate/strong to mild pain.

\section{fMRI results}

During acute pain stimulation, robust BOLD signals were seen in cortical regions typically involved in pain processing. These areas included the prefrontal cortices, ACC (anterior, medium, and dorsal), insula, thalamus, and PAG. Figure 4 shows corresponding group activation maps during acute thermal pain.

Brain pain activation at baseline for both sessions was similar, making these groups comparable at the beginning of the sessions and making results methodologically reliable. Corresponding Talairach coordinates, $t$-scores, cluster sizes, and Broadmann areas are given in detail in Table 1.

Overall diminution between T0 and T100 was significantly different between placebo and APAP ( $\beta=0.89$ vs $-0.032, P=0.001)$. Comparisons between the APAP

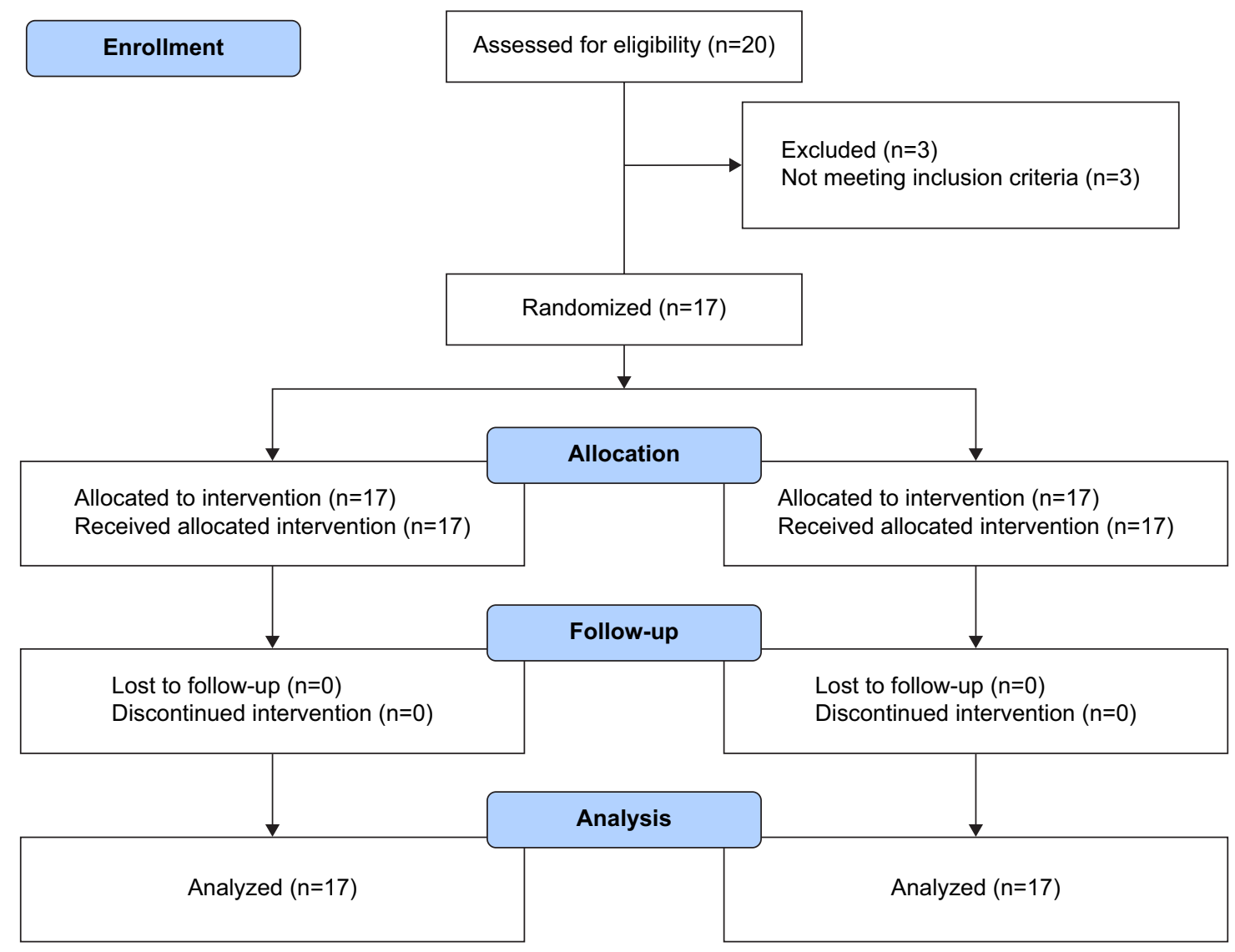

Figure 3 Flowchart of the study. 


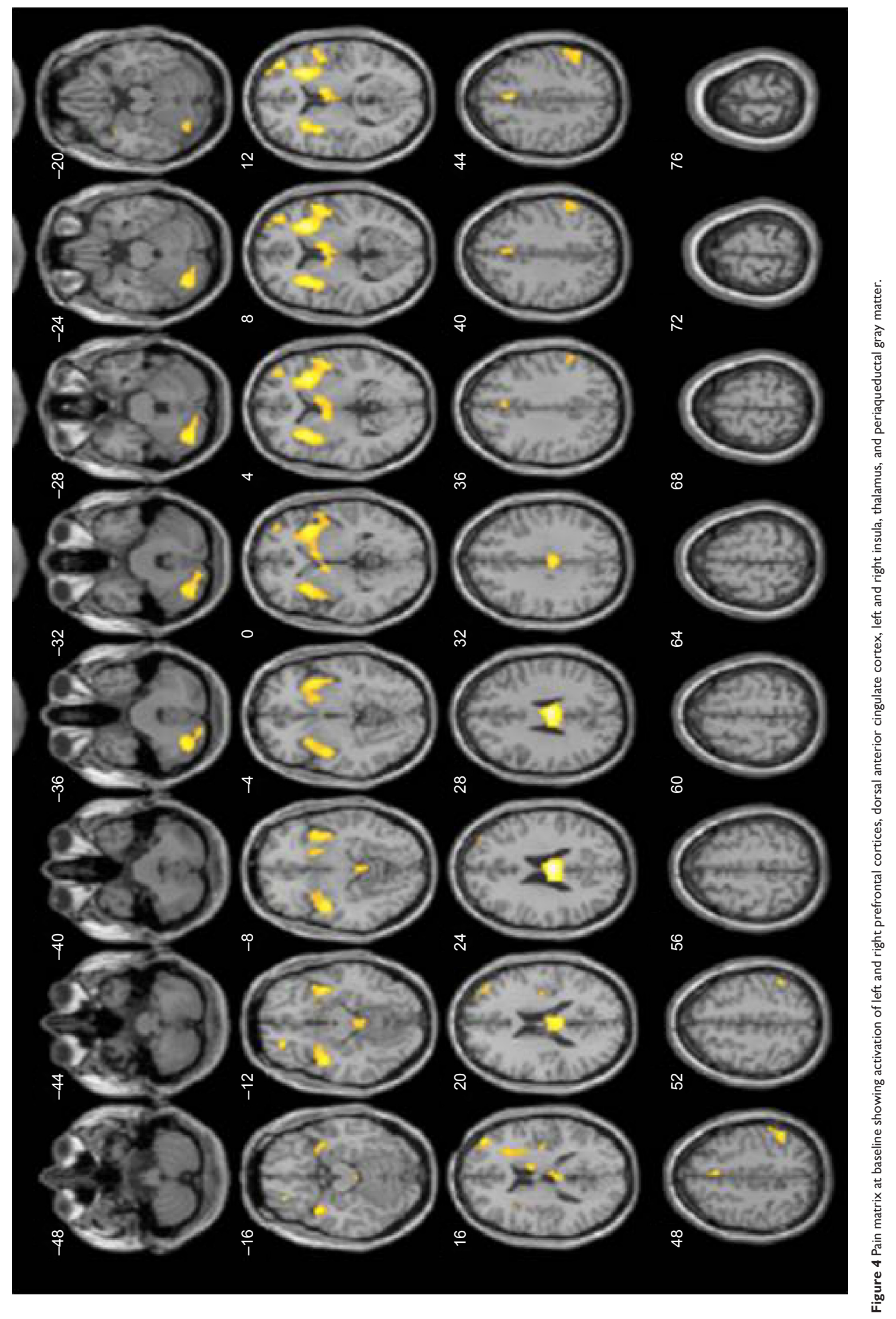


Table I Coordinates of the regions of interest

\begin{tabular}{llll}
\hline Region & \multicolumn{2}{l}{ TAL coordinates } \\
\cline { 2 - 4 } & $\mathbf{X}$ & $\mathbf{Y}$ & $\mathbf{Z}$ \\
\hline $\begin{array}{l}\text { Cingulate gyrus } \\
\text { Anterior }\end{array}$ & -12 & 20 & 40 \\
$\quad$ Middle & 10 & 8 & 42 \\
$\quad \begin{array}{l}\text { Posterior } \\
\text { Prefrontal cortex }\end{array}$ & 16 & -20 & 38 \\
$\quad$ Left & & & \\
$\quad$ ROI I & -20 & 56 & 10 \\
$\quad$ ROI 2 & -30 & 54 & -8 \\
Right & & & \\
$\quad$ ROI I & 30 & 46 & -14 \\
$\quad$ ROI 2 & 48 & 36 & -12 \\
Insula & & & \\
Left & -36 & -2 & 0 \\
Right & 38 & -8 & 6 \\
PAG & 4 & -36 & -10 \\
Thalamus & & & 11 \\
Left & -16 & -17 & 10 \\
Right & 18 & -16 & \\
\hline
\end{tabular}

Abbreviations: $\mathrm{fMRI}$, functional magnetic resonance imaging; PAG, periaqueductal gray matter; ROI, regions-of-interest; TAL, Talairach coordinates.

and placebo groups showed that significant diminished activations were observed for APAP at T100 in prefrontal cortices ( $P=0.006$ and 0.002 left and right, respectively), ACC $(P=0.02,0.004$, and 0.002 for anterior, medium, and dorsal, respectively), insula $(P=0.001$ and $P<0.001$ left and right, respectively) and thalami ( $P=0.007$ and 0.003 for left and right, respectively). The diminution was not significant in the PAG $(P=0.54)$, Figure 5. The correlation between fMRI signal for APAP diminution of activation (T100-T0) and the diminution of pain intensity was significant for $\operatorname{APAP}(P=0.002)$ and not for placebo. The imaging results are therefore consistent with the behavioral analgesic effects of APAP.

\section{Discussion}

This trial, for the first time, visualizes the central effect of APAP in antinociception through neuroimaging. The study provides evidence in healthy subjects that APAP reduces the pain-related BOLD signal responses arising from peripheral noxious thermal stimulation, in several brain areas of the pain matrix. However, a limitation of the study is that it does not allow to differentiate between a peripheral and a central mechanism of action as both could induce changes in the pain matrix. The reduction of perceived pain intensity scores simultaneous with the reduction in pain-related activity is consistent with the observed changes in BOLD signal resulting from the analgesic effect of APAP. This point is important as an analgesic treatment may as part of its primary effect reduce or increase the BOLD signal by reducing or increasing neuronal activity/ local metabolic consumption. ${ }^{21}$ Compared to placebo, APAP significantly reduces the pain-related BOLD signal responses arising from the noxious thermal stimulation in the selected ROI, insula, ACC, thalamus, and prefrontal cortices. This suggests an inhibitory effect of APAP on spinothalamic tracts leading to a decreased activation of higher structures and a resulting anti-nociceptive effect of APAP. Some of these brain areas, dorsal ACC, and insula are also described to be involved in psychological and cognitive-emotional processes. ${ }^{16,26} \mathrm{An}$ fMRI study ${ }^{38}$ found that APAP reduced neural responses to social rejection in these brain regions, demonstrating an overlap between social and physical pain. A recent cluster trial has also shown that APAP significantly reduces agitation and behavioral or psychological symptoms in patients with communication disorders with a beneficial effect on well-being, with a probable impact on physical pain but also on other components of pain. ${ }^{39}$

This study also demonstrates some degree of deactivation of the PAG with APAP but does not reach the level of significance set in our study. The PAG is known to have a pivotal role in the descending pain modulatory pain system ${ }^{17-40,41}$ with top-down influences between higher centers from the cingulo-frontal cortex to the PAG and RVM downward to the spinal cord. The PAG is also an important projection site of ascending pain pathways that might have a role in pain modulation distinct from descending projections in the spinal cord. ${ }^{42}$ We suggest a number of hypotheses to explain our findings. Firstly, a covariation between the activity in the dorsal ACC and the PAG has been demonstrated in placebo analgesia and opioid analgesia, leading to opioid release within the brain stem. ${ }^{37}$ White matter tracks between ACC and PAG have also been described suggesting an anatomical support to the link between ACC and PAG, ${ }^{43}$ and neuropharmacological evidence has suggested that corticobulbar neurons in the ACC directly or indirectly excite the PAG. ${ }^{9}$ Our findings confirm a sort of continuum in APAP effect from higher centers to the PAG but with lesser impact than has been observed with morphine. ${ }^{15}$ Considering that the ACC and the PAG are very rich in opiate receptors, a weak opioidergic effect of APAP cannot be discarded and this corroborates a number of preclinical and clinical studies, discussing contradictory findings on the implication of opioidergic mechanisms in APAP mechanism of action. A clinical study in healthy volunteers ${ }^{35}$ using APAP and naloxone with cerebral-evoked potential suggested a possible but weak effect of opioid mechanisms in this context. 

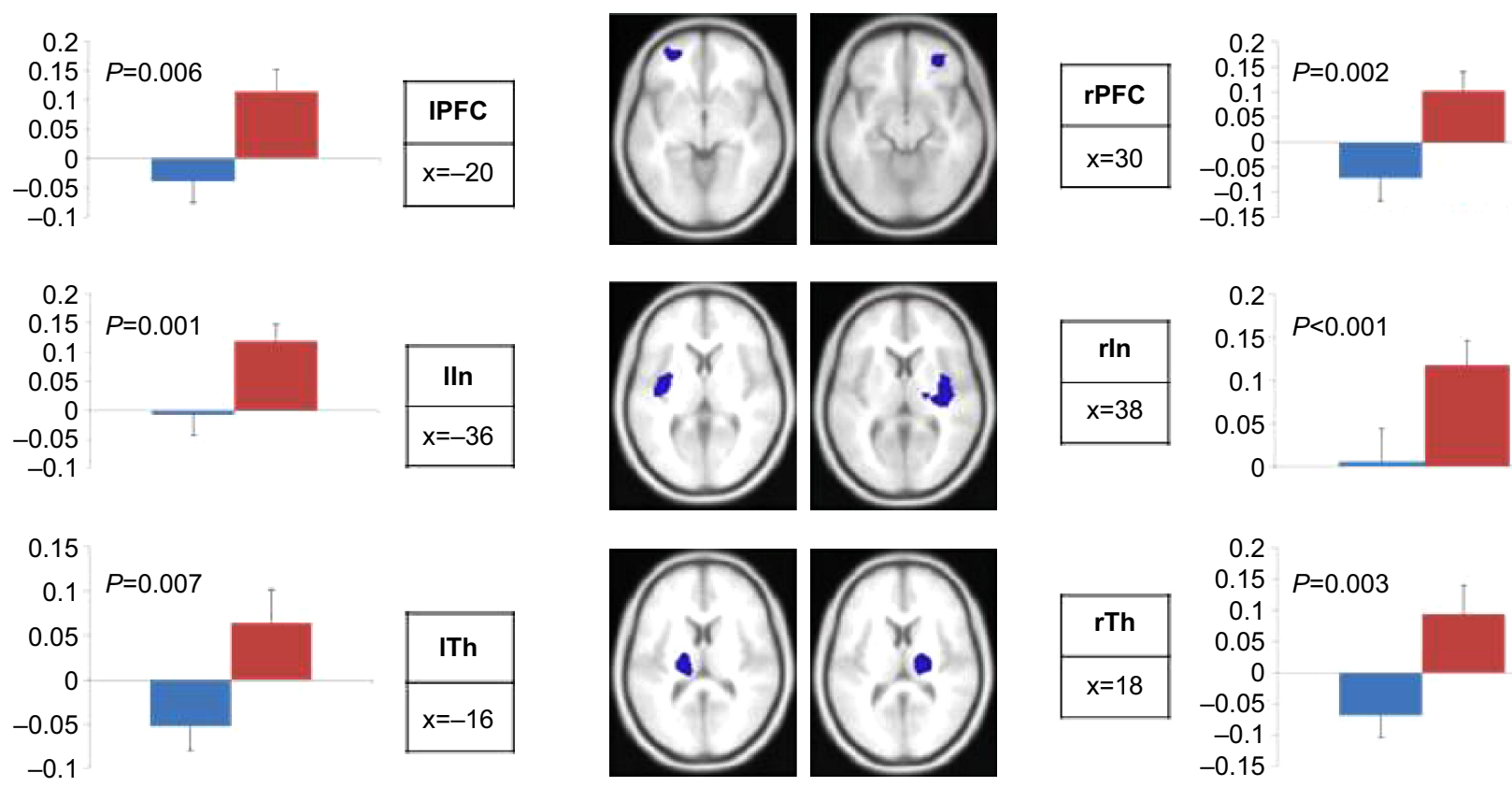

0.2

$0.15 P<0.001$
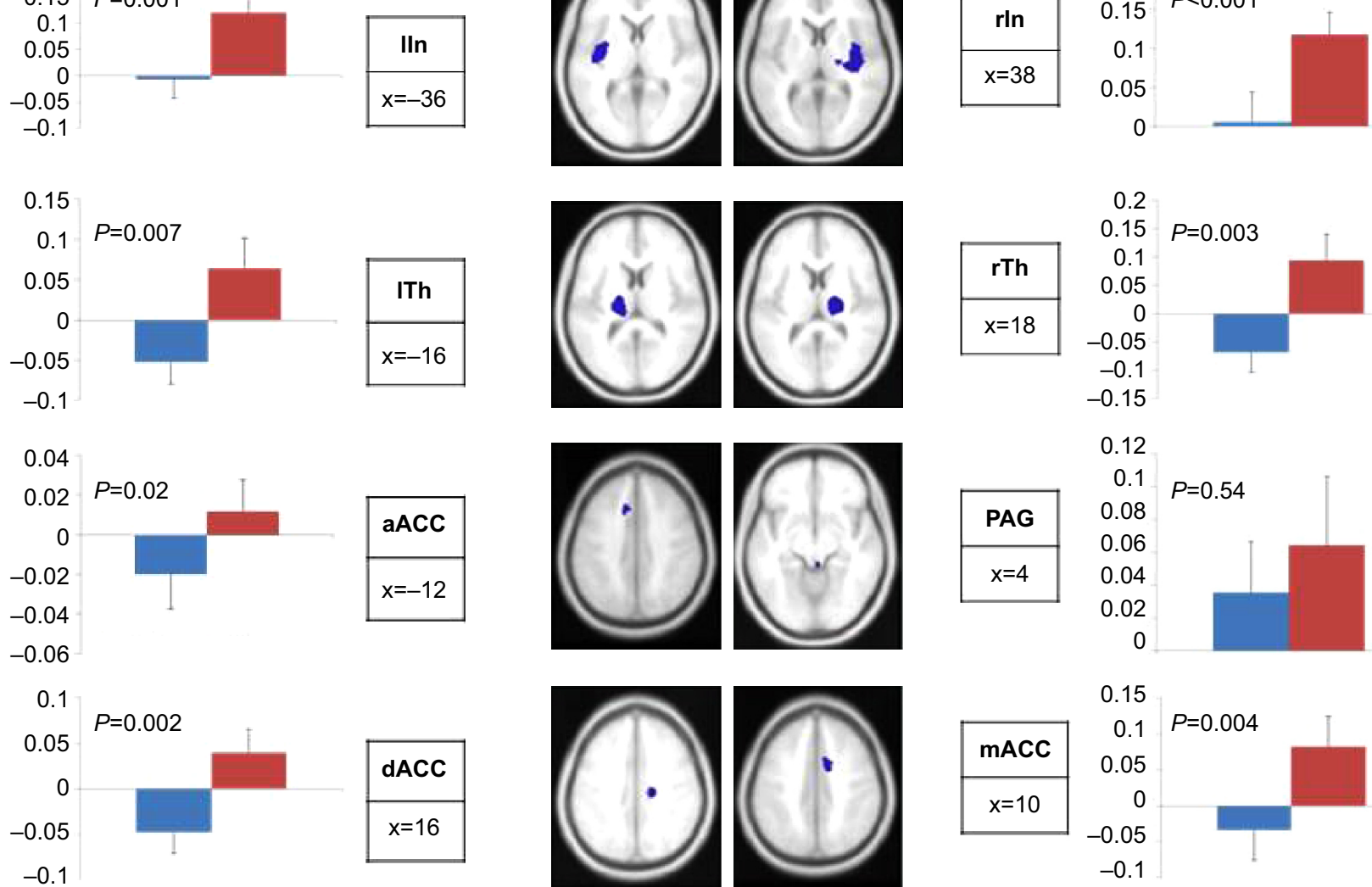
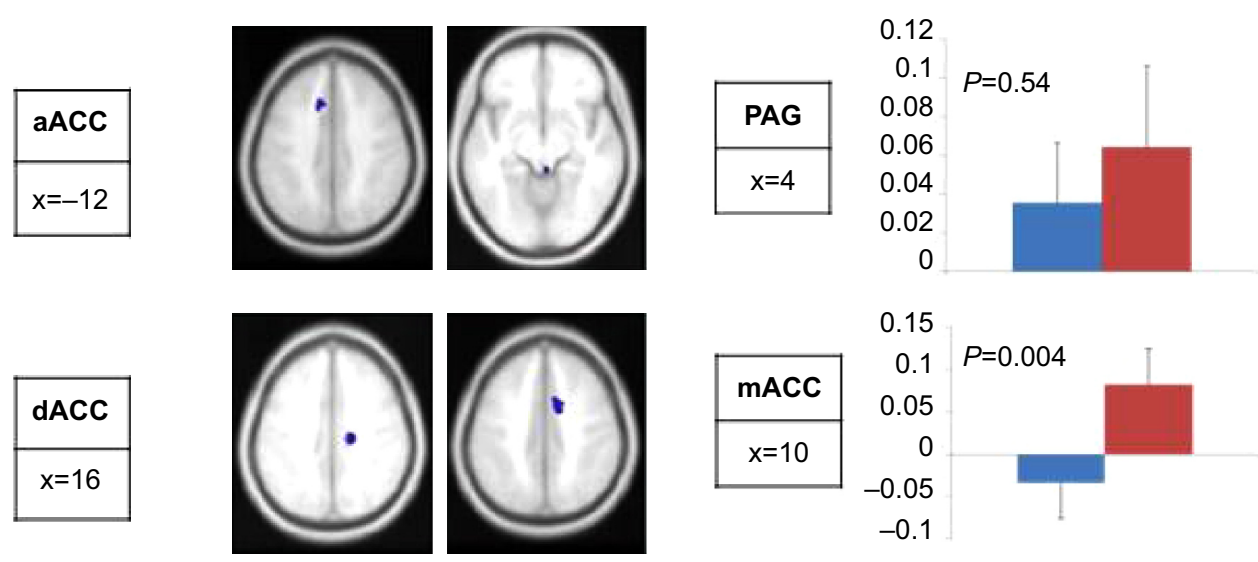

0.15

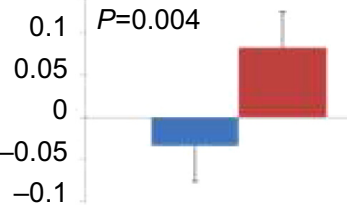

- APAP - Placebo

- APAP Placebo

Figure $5 \mathrm{ROI}$ analysis performed on physical pain regions revealed anti-nociceptive effect of APAP compared to placebo.

Notes: Bar graphs demonstrate the $\beta$-values of APAP deactivation ( $T+100$ minutes $>$ baseline) and placebo deactivation ( $T+100$ minutes $>$ baseline) extracted from each cluster. $\beta$-values associated with APAP differed significantly from the $\beta$-values associated with placebo in all regions (prefrontal cortices left and right [IPRC, rPFC], anterior cingulate cortex [ACC], anterior [a], medium [m], dorsal [d]), insula left and right (Iln, rln), and thalamus left and right (ITh, rTh) but in the periaqueductal gray matter (PAG).

Abbreviations: ROI, regions-of-interest; APAP, paracetamol.

Secondly, the PAGis in a physiological continuum with RVM neurons that have been classified as on, off, and neutral cells. We suggest that the maintained partial activation of the PAG enables release of neurotransmitters and endogenous opioids that exert their anti-nociceptive action through the synapses of the RVM system, a mechanism similar to the one shown for morphine. ${ }^{18,44}$ APAP might have a direct effect and activate PAG off cells and disinhibit RVM off cells or may activate PAG and RVM on cells to exert their anti-nociceptive effect on dorsal horn transmission neurons. One of the key neurotransmitters involved directly or indirectly in APAP mechanism of action is serotonin ${ }^{4,11,12}$ and serotonin receptors located at brain stem level, in combination with other mediators, ${ }^{2,11}$ play a role in the analgesic effect of APAP. Spinal serotonin receptors contribute to descending anti-nociceptive influence induced by stimulation of the PAG. ${ }^{45,46}$ An RVM subpopulation of neutral cells is serotoninergic, has more variable effects, and possibly modulates the effects induced by on and off cells to contribute to descending inhibitory controls relaying through the activation of PAG and RVM. ${ }^{18}$ This may be a specificity of action of APAP, as serotonergic neurotransmission, which recruits many serotonergic receptors including 5-HT2A and $5-\mathrm{HT} 2 \mathrm{C}^{47}$ in the PAG plays a critical role in the elaboration of antinociception and a link between spinal 5-HT(7) and A1 receptors in the spinal cord has been shown to be relevant to antinociception by systemic APAP. ${ }^{48}$ 
Thirdly, the stimulus we used was 3 degrees above pain threshold and corresponds to moderate to severe pain, rated as $6 / 10$ at baseline. APAP is recommended for mild to moderate pain (3 to 7) but in severe or moderate/severe pain, opiates should then be prescribed. Although the subjects report less pain after APAP, there is some residual pain $(4 \pm 2)$ that may explain that $\mathrm{PAG}$ activation is partially maintained.

Finally, cortical areas that are not included in the pain matrix, other brain stem regions, parallel descending pain inhibitory pathways may also be involved in APAP mechanism of action, and bidirectional links between the brain stem and cortical areas have also been described. Connectivity studies and pharmacological trials using tagged 5HT antagonists ${ }^{4}$ would help to decipher the implication of the serotonergic system in APAP analgesic effect. Reports show that APAP easily passes the blood-brain barrier, that the enzyme involved in AM404 synthesis (FAAH), as well as vanilloid and cannabinoid receptors and neuronal calcium channels are all widely distributed among supraspinal and spinal structures ${ }^{7-13}$ emphasizing the need for central studies. Considering the recent observation that a single APAP dose may increase the transcription of efflux transporter genes such as $P$ - $g p$ at the blood-brain barrier and enhance their production in the course of 3 hours, ${ }^{49}$ a study with repeated doses of APAP would show if longer term impaired brain permeation may have consequences on neuroimaging of pain with APAP, and may even decrease the effect of APAP, as suggested in a recent study challenging the international recommendations of APAP in osteoarthritis. ${ }^{50}$

\section{Conclusion}

This trial visualizes for the first time by fMRI the supraspinal effect of APAP after a peripheral stimulation. It shows the decreased activation of a number of brain structures involved in physical pain and in cognitive/emotional domains of pain. Linking the findings of this study to the mechanistic cascade of events described in the literature, we suggest a top-down phenomenon with an active engagement of the PAG for APAP mechanism of action and a specific action on the midbrain neurotransmission system. Further binding and connectivity studies are needed to evaluate how the analgesic effect of APAP relates to cerebral and descending modulation of pain, especially in chronic administration in patients.

\section{Acknowledgments}

To Clermont-Ferrand University Hospital for providing full financial support of the trial. SL and RV received funding from the French program "Investissement d'Avenir" run by the "Agence Nationale pour la Recherche"; grants "IHU-A-ICM, Paris Institute of Translational neuroscience" and "Infrastructure d'avenir en Biologie Santé - ANR-11INBS-0006".

\section{Disclosure}

The authors declare no conflicts of interest in this work.

\section{References}

1. Alloui A, Pelissier T, Dubray C, Lavarenne J, Eschalier A. Tropisetron inhibits the antinociceptive effect of intrathecally administered acetaminophen and serotonin. Fundam Clin Pharmacol. 1996;10(4): 406-407.

2. Graham GG, Davies MJ, Day RO, Mohamudally A, Scott KF. The modern pharmacology of paracetamol: therapeutic actions, mechanism of action, metabolism, toxicity and recent pharmacological findings. Inflammopharmacology. 2013;21(3):201-232.

3. Pickering G, Estève V, Loriot MA, Eschalier A, Dubray C. Acetaminophen reinforces descending inhibitory pain pathways. Clin Pharmacol Ther. 2008;84(1):47-51.

4. Pickering G, Loriot MA, Libert F, Eschalier A, Beaune P, Dubray C. Acetaminophen: First evidence of a central serotonergic mechanism of action in humans. Clin Pharmacol Ther. 2006;79(4):371-378.

5. Andersson DA, Gentry C, Alenmyr L, et al. TRPA1 mediates spinal antinociception induced by acetaminophen and the cannabinoid $\Delta(9)$ tetrahydrocannabiorcol. Nat Commun. 2011;2:551.

6. Högestätt ED, Jönsson BA, Ermund A, et al. Conversion of acetaminophen to the bioactive $\mathrm{N}$-acylphenolamine AM404 via fatty acid amide hydrolase-dependent arachidonic acid conjugation in the nervous system. J Biol Chem. 2005;280(36):31405-31412.

7. Barrière DA, Mallet C, Blomgren A, et al. Fatty acid amide hydrolasedependent generation of antinociceptive drug metabolites acting on TRPV1 in the brain. PLoS One. 2013;5;8(8):e70690.

8. Bonnefont J, Daulhac L, Etienne M, et al. Paracetamol recruits spinal p42/p44 MAPKs and GH/IGF-1 receptors to produce analgesia via the serotonergic system. Mol Pharmacol. 2007;71(2):407-415.

9. Kerckhove N, Mallet C, François A, et al. Ca(v)3.2 calcium channels: the key protagonist in the supraspinal effect of paracetamol. Pain. 2014; 155(4):764-772.

10. Mallet C, Barriere DA, Ermund A, Jönsson BA, Eschalier A, et al. TRPV(1) in brain is involved in acetaminophen-induced antinociception. PLoS One. 2010;5(9):e12748.

11. Mallet C, Daulhac L, Bonnefont J, et al. Endocannabinoid and serotonergic systems are needed for acetaminophen-induced analgesia. Pain. 2008;139(1):190-200.

12. Pini LA, Sandrini M, Vitale G. The antinociceptive action of paracetamol is associated with changes in the serotonergic system in the rat brain. Eur J Pharmacol. 1996;308(1):31-40.

13. Zygmunt PM, Chuang H, Movahed P, Julius D, Hogestatt ED. The anandamide transport inhibitor AM404 activates vanilloid receptors. Eur J Pharmacol. 2000;396(1):39-42.

14. Hadjipavlou G, Dunckley P, Behrens TE, Tracey I. Determining anatomical connectivities between cortical and brainstem pain processing regions in humans: a diffusion tensor imaging study in healthy controls. Pain. 2006;123(1-2):169-78.

15. Millan MJ. Descending control of pain. Prog Neurobiol. 2002;66(6) 355-374.

16. Tracey I, Mantyh PW. The cerebral signature for pain perception and its modulation. Neuron. 2007;55(3):377-391.

17. Basbaum AI, Fields HL. Endogenous pain control systems: brainstem spinal pathways and endorphin circuitry. Annu Rev Neurosci. 1984; 7 : 309-338.

18. Fields HL, Basbaum AI. Central nervous system mechanisms of pain modulation. In: Melzack R, Wall PD, editors. Textbook of pain. London: Churchill Livingstone; 2005:125-142. 
19. Baliki MN, Geha PY, Fields HL, Apkarian AV. Predicting value of pain and analgesia: nucleus accumbens response to noxious stimuli changes in the presence of chronic pain. Neuron. 2010;66(1):149-160.

20. Upadhyay J, Anderson J, Schwarz AJ, et al. Imaging Drugs With and Without Clinical Analgesic Efficacy. Neuropsychopharmacology. 2011; 36(13):2659-2673.

21. Wise RG, Williams P, Tracey I. Using fMRI to quantify the time dependence of remifentanil analgesia in the human brain. Neuropsychopharmacology. 2004;29(3):626-635.

22. Apkarian AV, Bushnell MC, Treede RD, Zubieta JK. Human brain mechanisms of pain perception and regulation in health and disease. Eur J Pain. 2005;9(4):463-484.

23. Peyron R, Faillenot I. Imagerie fonctionnelle cérébrale appliquée à l'analyse des phénomènes douloureux. [Functional brain mapping of pain perception]. Med Sci (Paris). 2011;27(1):82-87. French.

24. Rainville P, Duncan GH, Price DD, Carrier B, Bushnell MC. Pain affect encoded in human anterior cingulate but not somatosensory cortex. Science. 1997;277(5328):968-971.

25. Eisenberger NI, Lieberman MD. Why rejection hurts: a common neural alarm system for physical and social pain. Trends Cogn Sci. 2004;8(7): 294-300.

26. Kross E, Berman MG, Mischel W, Smith EE, Wager TD. Social rejection shares somatosensory representations with physical pain. Proc Natl Acad Sci U S A. 2011;108(15):6270-6275.

27. Becerra L, Harter K, Gonzalez RG, Borsook D. Functional magnetic resonance imaging measures of the effects of morphine on central nervous system circuitry in opioid-naive healthy volunteers. Anesth Analg. 2006;103(1):208-216.

28. Gear R, Becerra L, Upadhyay J, et al. Pain facilitation brain regions activated by nalbuphine are revealed by pharmacological fMRI. PLoS One. 2013;8(1):e50169.

29. Leppa M, Korvenoja A, Carlson S, et al. Acute opioid effects on human brain as revealed by functional magnetic resonance imaging. Neuroimage. 2006;31(2):661-669.

30. Oertel BG, Preibisch C, Wallenhorst T, et al. Differential opioid action on sensory and affective cerebral pain processing. Clin Pharmacol Ther. 2008;83(4):577-588.

31. Upadhyay J, Anderson J, Baumgartner R, et al. Modulation of CNS pain circuitry by intravenous and sublingual doses of buprenorphine. Neuroimage. 2012;59(4):3762-3773.

32. Wise RG, Rogers R, Painter D, et al. Combining fMRI with a pharmacokinetic model to determine which brain areas activated by painful stimulation are specifically modulated by remifentanil. Neuroimage. 2002;16(4):999-1014.

33. Maihöfner C, Ringler R, Herrndobler F, Koppert W. Brain imaging of analgesic and antihyperalgesic effects of cyclooxygenase inhibition in an experimental human pain model: a functional MRI study. Eur $J$ Neurosci. 2007;26(5):1344-1356.

34. Rogers R, Wise RG, Painter DJ, Longe SE, Tracey I. An investigation to dissociate the analgesic and anesthetic properties of ketamine using functional magnetic resonance imaging. Anesthesiology. 2004;100(2): 292-301.

35. Pickering G, Moustafa F, Desbrandes S, Cardot JM, Roux D, Dubray C. Paracetamol and opioid pathways: a pilot randomised clinical trial. Fund Clin Pharmacol. 2013;27(3):339-345.
36. Granovsky Y, Matre D, Sokolik A, Lorenz J, Casey KL. Thermoreceptive innervation of human glabrous and hairy skin: a contact heat evoked potential analysis. Pain. 2005;115(3):238-247.

37. Petrovic $P$, Kalso E, Petersson KM, Ingvar M. Placebo and opioid analgesia? imaging a shared neuronal network. Science. 2002;295(5560): $1737-1740$.

38. Dewall CN, Macdonald G, Webster GD, et al. Acetaminophen reduces social pain: behavioral and neural evidence. Psychol Sci. 2010;21(7): 931-937.

39. Husebo BS, Ballard C, Sandvik R, Nilsen OB, Aarsland D. Efficacy of treating pain to reduce behavioural disturbances in residents of nursing homes with dementia: cluster randomised clinical trial. BMJ. 2011;343: d4065.

40. La Cesa S, Tinelli E, Toschi N, et al. fMRI pain activation in the periaqueductal gray in healthy volunteers during the cold pressor test. Magn Reson Imaging. 2014;32(3):236-240.

41. Linnman C, Moulton EA, Barmettler G, Becerra L, Borsook D. Neuroimaging of the periaqueductal gray: state of the field. Neuroimage. 2012;60(1):505-522.

42. Yoshida W, Seymour B, Koltzenburg M, Dolan RJ. Uncertainty increases pain: evidence for a novel mechanism of pain modulation involving the periaqueductal gray. J Neurosci. 2013;33(13):5638-5646.

43. Mantyh PW. Forebrain projections to the periaqueductal gray in the monkey, with observations in the cat and rat. J Comp Neurol. 1982;206(2): 146-158.

44. Tracey I, Ploghaus A, Gati JS, et al. Imaging attentional modulation of pain in the periaqueductal gray in humans. J Neurosci. 2002;22(7): $2748-2752$.

45. Aimone LD, Jones SL, Gebhart GF. Stimulation-produced descending inhibition from the periaqueductal gray and nucleus raphe magnus in the rat: mediation by spinal monoamines but not opioids. Pain. 1987;31(1): 123-136.

46. Rivot JP, Pointis D, Besson JM. A comparison of the effects of morphine on 5-HT metabolism in the periaqueductal gray, ventromedial medulla and medullary dorsal horn: in vivo electrochemical studies in freely moving rats. Brain Res. 1989;495(1):140-144. Available from: http://www.ncbi.nlm.nih.gov/pubmed/2776031

47. de Freitas RL, de Oliveira RC, de Oliveira R, Paschoalin-Maurin T, de Aguiar Corrêa FM, Coimbra NC. The role of dorsomedial and ventrolateral columns of the periaqueductal gray matter and in situ 5- $\mathrm{HT}_{2} \mathrm{~A}$ and 5- $\mathrm{HT}_{2} \mathrm{C}$ serotonergic receptors in post-ictal antinociception. Synapse. 2014;68(1):16-30.

48. Liu J, Reid AR, Sawynok J. Antinociception by systemicallyadministered acetaminophen (paracetamol) involves spinal serotonin 5-HT7 and adenosine A1 receptors, as well as peripheral adenosine A1 receptors. Neurosci Lett. 2013;536:64-68.

49. Slosky LM, Thompson BJ, Sanchez-Covarrubias L, et al. Acetaminophen modulates P-glycoprotein functional expression at the blood-brain barrier by a constitutive androstane receptor-dependent mechanism. Mol Pharmacol. 2013;84(5):774-786.

50. Williams WC, Maher CG, McLachlan AJ, Hancock MJ, Day RO, ChungWei CL. Efficacy of paracetamol for acute low-back pain: a double-blind, randomised controlled trial. Lancet. 2014;384(9954):1586-1596.
Drug Design, Development and Therapy

\section{Publish your work in this journal}

Drug Design, Development and Therapy is an international, peerreviewed open-access journal that spans the spectrum of drug design and development through to clinical applications. Clinical outcomes, patient safety, and programs for the development and effective, safe, and sustained use of medicines are a feature of the journal, which

\section{Dovepress}

has also been accepted for indexing on PubMed Central. The manuscript management system is completely online and includes a very quick and fair peer-review system, which is all easy to use. Visit http://www.dovepress.com/testimonials.php to read real quotes from published authors. 